Homology, Homotopy and Applications, vol.15(1), 2013, pp.73-82

\title{
ON SEMISIMPLICIAL SETS SATISFYING THE KAN CONDITION
}

\author{
JAMES E. MCCLURE
}

\author{
(communicated by Daniel Dugger)
}

\begin{abstract}
A semisimplicial set has face maps but not degeneracies. A basic fact, due to Rourke and Sanderson, is that a semisimplicial set satisfying the Kan condition can be given a simplicial structure. The present paper gives a combinatorial proof of this fact and a generalization to multisemisimplicial sets.
\end{abstract}

\section{Introduction}

A semisimplicial set $X$ is a sequence of sets $X_{n}$ for $n \geqslant 0$ with maps $d_{i}: X_{n} \rightarrow X_{n-1}$ for $0 \leqslant i \leqslant n$ satisfying

$$
d_{i} d_{j}=d_{j-1} d_{i} \quad \text { if } i<j .
$$

Elements of $X_{n}$ are called $n$-simplices, and the maps $d_{i}$ are called face maps.

Semisimplicial sets occur in various areas of mathematics, especially geometric topology, surgery theory (e.g., [3]), and homological algebra (e.g., $[\mathbf{6}, \mathbf{5}])$.

Definition 1.1. A semisimplicial set $X$ satisfies the Kan condition if, for every collection of $n+1 n$-simplices $x_{0}, \ldots, x_{k-1}, x_{k+1}, \ldots, x_{n+1}$ satisfying

$$
d_{i} x_{j}=d_{j-1} x_{i} \quad \text { whenever } i<j \text { with } i \neq k \neq j,
$$

there is an $n+1$ simplex $x$ with $d_{i} x=x_{i}$ for all $i \neq k$.

In [4], Rourke and Sanderson used PL topology to prove that a semisimplicial set which satisfies the Kan condition has a simplicial structure:

Theorem 1.2. [4, Theorem 5.7] Let $X$ be a semisimplicial set satisfying the Kan condition. Then there are functions

$$
s_{j}: X_{n} \rightarrow X_{n+1},
$$

for $n \geqslant 0$ and $0 \leqslant j \leqslant n$, with the following properties.

$$
\begin{array}{rlrl}
d_{i} s_{j} & =s_{j-1} d_{i} & & \text { if } i<j . \\
d_{i} s_{j} x=x & & \text { if } i=j, j+1 . \\
d_{i} s_{j}=s_{j} d_{i-1} & & \text { if } i>j+1 . \\
s_{j} s_{i}=s_{i} s_{j-1} & & \text { if } i<j .
\end{array}
$$

The author was partially supported by NSF grants. He thanks the Lord for making his work possible. Received October 19, 2012, revised November 17, 2012; published on March 6, 2013. 2000 Mathematics Subject Classification: Primary 55P43; Secondary 57R67, 57P10.

Key words and phrases: semisimplicial set, Kan condition.

Article available at http://intlpress.com/HHA/v15/n1/a4 and doi:10.4310/HHA.2013.v15.n1.a4 Copyright (C) 2013, International Press. Permission to copy for private use granted. 
Note that (5) is written in a slightly nonstandard form which is equivalent to the usual one. Also note that the theorem does not say that the simplicial structure on $X$ is unique, nor does it give a functor from Kan semisimplicial sets to simplicial sets.

The purpose of this note is to generalize Theorem 1.2 to multisemisimplicial sets, for use in [2]. It is not at all clear how to generalize the geometric argument given by Rourke and Sanderson, so instead I will give a combinatorial proof of Theorem 1.2 which generalizes easily to the multisemisimplicial setting.

The organization of the paper is as follows. The proof of Theorem 1.2 is a double induction which is carried out in Sections 2 and 3. An auxiliary lemma is proved in Section 4. The generalization of Theorem 1.2 to multisemisimplicial sets is stated in Section 5 and proved in Sections 6-8. Section 9 gives an application of Theorem 1.2, using it to give a new proof of [4, Corollary 5.4].

I would like to thank Stefan Schwede for a helpful conversation.

A note on terminology. The first appearance of semisimplicial sets in the literature was in $[\mathbf{1}]$, where they were called semisimplicial complexes. The motivation for the name is that an ordered simplicial complex has two properties: (i) there are face maps satisfying (1) and (ii) a simplex is determined by its faces; a "semisimplicial complex" has only the first property. The same paper introduced the concept of "complete semisimplicial complexes," in which the word "complete" referred to the presence of degeneracy maps. During the 1950's and 1960's it became common to drop the word "complete" from the terminology (probably because all of the known applications required degeneracies) and to use the term semisimplicial complex to mean what is now called a simplicial set.

Semisimplicial complexes in the original sense were resurrected and renamed (as $\Delta$-sets) in [4]. The name $\Delta$-set seems infelicitous because the category that governs simplicial sets is called $\Delta$. In using the term semisimplicial set I am following the terminology of [6, Definition 8.1.9].

\section{Beginning of the proof of Theorem 1.2}

We will construct the degeneracies $s_{j} x$ by a double induction on $\operatorname{deg}(x)$ and $j$. Specifically, given $n \geqslant 0$ and $0 \leqslant k \leqslant n$, we assume that $s_{j} x$ has been constructed when $\operatorname{deg}(x)<n$ and also when $\operatorname{deg}(x)=n$ and $j<k$, and that properties (2)-(5) hold in all relevant cases (that is, in all cases involving only degeneracies that have already been constructed). Let $x \in X_{n}$; we need to construct $s_{k} x$.

There are two cases. The easier case (which does not use the Kan condition) is when $x$ is in the image of $s_{j}$ for some $j<k$. We give the proof for this case in this section.

Choose the smallest $j$ for which $x$ is in the image of $s_{j}$; then

$$
x=s_{j} w
$$

for some $w$. In fact this $w$ is unique, by the following lemma.

Lemma 2.1. Degeneracy maps are monomorphisms.

Proof. This is immediate from (3). 
We now define $s_{k} x$ to be $s_{j} s_{k-1} w$ (as required by (5)).

It remains to verify (2)-(5). The verifications of (2)-(4) are routine applications of the simplicial identities and are left to the reader.

For (5) we need a well-known fact:

Lemma 2.2. If $s_{j} w=s_{i} y$ for some $i$ with $j<i<k$ then there is a $v$ with $y=s_{j} v$ and $w=s_{i-1} v$.

Proof. Let $v=d_{j} y$. Then

$$
s_{i} y=s_{j} w=s_{j} d_{j} s_{j} w=s_{j} d_{j} s_{i} y=s_{j} s_{i-1} d_{j} y=s_{j} s_{i-1} v=s_{i} s_{j} v,
$$

so $y=s_{j} v$ by Lemma 2.1. Now

$$
s_{j} w=s_{i} y=s_{i} s_{j} v=s_{j} s_{i-1} v
$$

so $w=s_{i-1} v$ by Lemma 2.1 .

Now we verify (5). Let $y \in X_{n-1}$ and let $i<k$. Choose the smallest $j$ for which $s_{i} y$ is in the image of $s_{j}$. If $j=i$ we are done, otherwise let $s_{i} y=s_{j} w$. Let $v$ be the element given by Lemma 2.2. Then

$$
\begin{aligned}
s_{k} s_{i} y & =s_{j} s_{k-1} w \quad \text { by definition of } s_{k} \\
& =s_{j} s_{k-1} s_{i-1} v=s_{j} s_{i-1} s_{k-2} v=s_{i} s_{j} s_{k-2} v \\
& =s_{i} s_{k-1} s_{j} v \quad \text { because } j<i<k \text { so } j<k-1 \\
& =s_{i} s_{k-1} y,
\end{aligned}
$$

as required.

\section{Conclusion of the proof of Theorem $\mathbf{1 . 2}$}

Next we must construct $s_{k} x$ in the remaining case, when $x$ is not in the image of any $s_{j}$ with $j<k$.

First note that the simplicial identities determine all faces of $s_{k} x$, so it is not possible to build $s_{k} x$ directly from the Kan condition. Instead, we apply the Kan condition twice to construct a suitable element $T_{k} x$ in degree $\operatorname{deg}(x)+2$ and then define

$$
s_{k} x=d_{0} T_{k} x
$$

We will construct the elements $T_{j} x$ by a double induction on $\operatorname{deg}(x)$ and $j$. In order to see what properties we want $T_{j}$ to have in the inductive hypothesis, we use a heuristic argument. For $i<j$, we want the simplicial identity (2) to hold. The left-hand side of (2) will be equal to $d_{0} d_{i+1} T_{j}$ (using (6) and $d_{i} d_{0}=d_{0} d_{i+1}$ ) and the right-hand side will be $d_{0} T_{j-1} d_{i}$. The simplest way for the two sides to be equal is to have $d_{i+1} T_{j}=T_{j-1} d_{i}$, which we rewrite as

$$
d_{i} T_{j}=T_{j-1} d_{i-1} \quad \text { if } 0<i<j+1 ;
$$

this is the first of the properties we want. Similarly, the simplicial identity (4) leads 
to the equation

$$
d_{i} T_{j}=T_{j} d_{i-2} \quad \text { if } i>j+2 .
$$

Finally, the simplicial identity (3) leads to two equations:

$$
d_{j+1} T_{j}=d_{j+2} T_{j} \quad \text { for all } j,
$$

and

$$
d_{0} d_{j+1} T_{j} x=x \text { for all } j \text { and } x .
$$

Lemma 3.1. Let $X$ be a semisimplicial set satisfying the Kan condition. Then there are functions

$$
T_{j}: X_{n} \rightarrow X_{n+2},
$$

for $n \geqslant 0$ and $0 \leqslant j \leqslant n$, satisfying (7)-(10).

The proof will be given in the next section. We can now finish the proof of Theorem 1.2. Given that $x$ is not in the image of $s_{j}$ for any $j<k$, define $s_{k} x$ by (6). The simplicial identity (2) follows at once from (7), and (4) follows from (8). To see that $d_{k} s_{k} x=x$ we use (10), and then $d_{k+1} s_{k} x=x$ follows from (9). The identity (5) is vacuous because of the assumption on $x$.

\section{Proof of Lemma 3.1}

To see how the construction of $T_{j}$ works it's helpful to begin with the case $\operatorname{deg}(x)=$ 0 and $j=0$. Since we want (9) and (10) to hold we must have $d_{1} T_{0} x=d_{2} T_{0} x$ and $d_{0} d_{1} T_{0} x=x$. The Kan condition gives an element $y$ of degree 1 with $d_{0} y=x$. A second application of the Kan condition gives $T_{0} x$ with $d_{1} T_{0} x=d_{2} T_{0} x=y$. Then (9) and (10) are immediate from the construction, and (7) and (8) are vacuous in this case.

Now assume that $T_{j} x$ has been constructed with properties (7)-(10) for $\operatorname{deg}(x)<n$ and also for $\operatorname{deg}(x)=n$ and $j<k$. Let $x \in X_{n}$; we need to construct $T_{k} x \in X_{n+2}$.

We begin by constructing an element $y$ of degree $n+1$ which will play the role of $d_{k+1} T_{k} x$. We want $d_{0} y$ to be $x$ because of (10). For $0<j<k+1$ we want $d_{j} y$ to be $d_{k} T_{k-1} d_{j-1} x$ because of (7), and for $j>k+1$ we want $d_{j} y$ to be $d_{k+1} T_{k} d_{j-1} x$ because of (8). In order to apply the Kan condition we need to show that these choices are consistent:

Lemma 4.1. Given $x \in X_{n}$, let

$$
y_{j}= \begin{cases}x & \text { if } j=0, \\ d_{k} T_{k-1} d_{j-1} x & \text { if } 0<j<k+1, \\ d_{k+1} T_{k} d_{j-1} x & \text { if } j>k+1 .\end{cases}
$$

Then

$$
d_{i} y_{j}=d_{j-1} y_{i} \quad \text { whenever } i<j \text { with } i \neq k+1 \neq j .
$$

Proof of Lemma 4.1. First suppose $i=0$. Then the right-hand side of (12) is equal to $d_{j-1} x$, and the left-hand side simplifies to $d_{j-1} x$ by (10).

Next suppose $0<i<j<k+1$. Both sides simplify to $d_{k-1} T_{k-2} d_{j-2} d_{i-1} x$ by (7). 
If $0<i<k+1<j$ then both sides simplify to $d_{k} T_{k-1} d_{j-2} d_{i-1} x$, using (7) on the left side and (8) on the right side.

Finally, if $k+1<i<j$ then both sides simplify to $d_{k+1} T_{k} d_{j-2} d_{i-1} x$ by (8).

Now we apply the Kan condition to construct $y \in X_{n+1}$ with $d_{j} y=y_{j}$ for $j \neq$ $k+1$.

Next we will use the Kan condition to construct $T_{k} x$. We choose $d_{k+1} T_{k} x$ to be the element $y$ that was just constructed, and because of (9) we also choose $d_{k+2} T_{k} x$ to be $y$. For $0<j<k+1$ we want $d_{j} T_{k} x$ to be $T_{k-1} d_{j-1} x$ because of (7), and for $j>k+2$ we want $d_{j} T_{k} x$ to be $T_{k} d_{j-2} x$ because of (8).

We need to check consistency:

Lemma 4.2. Let

$$
z_{j}= \begin{cases}T_{k-1} d_{j-1} x & \text { if } 0<j<k+1 \\ y & \text { if } j \text { is } k+1 \text { or } k+2 \\ T_{k} d_{j-2} x & \text { if } j>k+2\end{cases}
$$

Then

$$
d_{i} z_{j}=d_{j-1} z_{i} \quad \text { whenever } 0<i<j .
$$

Proof of Lemma 4.2. First suppose $j<k+1$. Both sides of (13) simplify to

$$
T_{k-2} d_{j-2} d_{i-1} x
$$

by (7).

If $j=k+1$ then the right side is $d_{k} T_{k-1} d_{i-1} x$, and the left side simplifies to this by $(11)$.

If $i<k+1$ and $j=k+2$ both sides simplify to $d_{k} T_{k-1} d_{i-1} x$, using (11) on the left and (9) on the right.

If $i<k+1$ and $j>k+2$ both sides simplify to $T_{k-1} d_{j-3} d_{i-1} x$, using (7) on the left and (8) on the right.

If $i=k+1$ and $j=k+2$ both sides are equal to $d_{k+1} y$.

If $i=k+2$ both sides simplify to $d_{k+1} T_{k} d_{j-2} x$, using (9) on the left and (11) on the right.

If $i>k+2$ both sides simplify to $T_{k} d_{j-3} d_{i-2} x$ using (8).

Now we apply the Kan condition to construct $T_{k} x$ with $d_{j} T_{k} x=z_{j}$ for $j \neq 0$. All parts of the inductive hypothesis are true by construction.

\section{The multisemisimplicial analogue of Theorem $\mathbf{1 . 2}$}

Definition 5.1. Let $\ell \geqslant 1$.

(i) An $\ell$-fold multi-index $\mathbf{n}$ is a sequence of nonnegative integers $n_{1}, \ldots, n_{\ell}$.

(ii) For $1 \leqslant p \leqslant \ell$ let $\mathbf{e}_{p}$ be the $\ell$-fold multi-index with 1 in the $p$-th position and 0 in all other positions.

(iii) Addition of $\ell$-fold multi-indices is degreewise addtion and similarly for subtraction. 
(iv) An $\ell$-fold multisemisimplicial set is a collection of sets $X_{\mathbf{n}}$ indexed by the $\ell$-fold multi-indices, with maps

$$
d_{i}^{p}: X_{\mathbf{n}} \rightarrow X_{\mathbf{n}-\mathbf{e}_{p}}
$$

for $1 \leqslant p \leqslant \ell$ and $0 \leqslant i \leqslant n_{p}$, such that

$$
\begin{array}{ll}
d_{i}^{p} d_{j}^{p}=d_{j-1}^{p} d_{i}^{p} & \text { when } i<j, \text { and } \\
d_{i}^{p} d_{j}^{q}=d_{j}^{q} d_{i}^{p} & \text { when } p \neq q .
\end{array}
$$

The Kan condition for multisemisimplicial sets is analogous to that for semisimplicial sets: a simplex can be constructed from a consistent choice of all but one of its faces. Here is the formal definition.

Definition 5.2. Let $X$ be an $\ell$-fold multisemisimplicial set. $X$ satisfies the Kan condition if, for every choice of a multi-index $\mathbf{n}$, a pair $(r, k)$ with $1 \leqslant r \leqslant \ell$ and $0 \leqslant m \leqslant n_{r}$, and elements $x_{i}^{p} \in X_{\mathbf{n}-\mathbf{e}_{p}}$ for $(p, i) \neq(r, k)$ satisfying

$$
d_{i}^{p} x_{j}^{p}=d_{j-1}^{p} x_{i}^{p} \quad \text { for } i<j
$$

and

$$
d_{i}^{p} x_{j}^{q}=d_{j}^{q} x_{i}^{p} \quad \text { for } p \neq q,
$$

there exists an element $x \in X_{\mathbf{n}}$ with $d_{i}^{p} x=x_{i}^{p}$ for $(p, i) \neq(r, k)$.

The analogue of Theorem 1.2 says that a multisemisimplicial set satisfying the Kan condition has a multisimplicial structure. Here is the formal statement.

Theorem 5.3. Let $X$ be an $\ell$-fold multisemisimplicial set satisfying the Kan condition. Then there are functions

$$
s_{j}^{q}: X_{\mathbf{n}} \rightarrow X_{\mathbf{n}+\mathbf{e}_{q}}
$$

for $0 \leqslant j \leqslant n_{q}$, with the following properties.

$$
\begin{aligned}
d_{i}^{q} s_{j}^{q} & =s_{j-1}^{q} d_{i}^{q} & & \text { if } i<j . \\
d_{i}^{q} s_{j}^{q} x & =x & & \text { if } i=j, j+1 . \\
d_{i}^{q} s_{j}^{q} & =s_{j}^{q} d_{i-1}^{q} & & \text { if } i>j+1 . \\
s_{j}^{q} s_{i}^{q} & =s_{i}^{q} s_{j-1}^{q} & & \text { if } i<j . \\
d_{i}^{p} s_{j}^{q} & =s_{j}^{q} d_{i}^{p} & & \text { whenever } p \neq q . \\
s_{i}^{p} s_{j}^{q} & =s_{j}^{q} s_{i}^{p} & & \text { whenever } p \neq q .
\end{aligned}
$$

Remark 5.4. Notice that to prove (19) it suffices (by symmetry) to prove that the equation holds for $p>q$.

The proof of Theorem 5.3, which is quite similar to that of Theorem 1.2, will occupy the rest of this section and the next three sections. For an element $x \in X_{\mathbf{n}}$, let us define the total degree $|x|$ to be $n_{1}+\cdots+n_{\ell}$. We will construct the degeneracies $s_{j}^{q}$ by a double induction on $|x|$ and the pair $(q, j)$; we order the pairs $(q, j)$ using the lexicographic order.

Let $m \geqslant 0,1 \leqslant r \leqslant \ell$ and $k \geqslant 0$. Assume that $s_{j}^{q} x$ has been constructed when $|x|<m$, and also also when $|x|$ is equal to $m$ and the pair $(q, j)$ is less than $(r, k)$ in 
the lexicographic order, and that properties (14)-(19) hold in all relevant cases (that is, in all cases involving only degeneracies that have already been constructed). Let $x \in X_{\mathbf{n}}$ with $n_{1}+\cdots+n_{\ell}=m$ and suppose $0 \leqslant k \leqslant n_{r}$; we need to construct $s_{k}^{r} x$.

There are three cases, which are dealt with in Sections 6-8:

Case $1 x$ is in the image of $s_{j}^{q}$ for some $q<r$.

Case $2 x$ is not in the image of $s_{j}^{q}$ for $q<r$, but it is in the image of $s_{j}^{r}$ for some $j<k$.

Case $3 x$ is not in the image of $s_{j}^{q}$ when $(q, j)<(r, k)$.

\section{Proof of Theorem 5.3: Case 1}

Let $(q, j)$ be the smallest pair with $x$ in the image of $s_{j}^{q}$; then

$$
x=s_{j}^{q} w
$$

for a unique $w$. Define

$$
s_{k}^{r} x=s_{j}^{q} s_{k}^{r} w
$$

(as required by (19)). We need to verify (14)-(19).

For property (14), both sides simplify to $s_{j}^{q} s_{k-1}^{r} d_{i}^{r} w$, using (18) and (14) on the left and (18) and (19) on the right. The verifications for (15) and (16) are similar.

For (17) we need:

Lemma 6.1. If $s_{j}^{q} w=s_{i}^{p} y$ with $p \neq q$ then there is a $v$ with $y=s_{j}^{q} v$ and $w=s_{i}^{p} v$.

Proof of Lemma 6.1. The proof is similar to that of Lemma 2.2. Let $v=d_{j}^{q} y$. Then

$$
s_{i}^{p} y=s_{j}^{q} w=s_{j}^{q} d_{j}^{q} s_{j}^{q} w=s_{j}^{q} d_{j}^{q} s_{i}^{p} y=s_{j}^{q} s_{i}^{p} d_{j}^{q} y=s_{j}^{q} s_{i}^{p} v=s_{i}^{p} s_{j}^{q} v,
$$

so $y=s_{j}^{q} v$. Now

$$
s_{j}^{q} w=s_{i}^{p} y=s_{i}^{p} s_{j}^{q} v=s_{j}^{q} s_{i}^{p} v
$$

so $w=s_{i}^{p} v$.

Now we verify (17). Let $y \in X_{\mathbf{n}-\mathbf{e}_{r}}$ and let $i<k$. Suppose that $s_{i}^{r} y$ is in the image of $s_{j}^{q}$ for some $q<r$, and choose the smallest such pair $(q, j)$; then $s_{i}^{r} y=s_{j}^{q} w$ for some $w$. Let $v$ be the element given by Lemma 6.1. Then

$$
\begin{aligned}
s_{k}^{r} s_{i}^{r} y & =s_{j}^{q} s_{k}^{r} w \quad \text { by definition of } s_{k}^{r} \\
& =s_{j}^{q} s_{k}^{r} s_{i}^{r} v=s_{j}^{q} s_{i}^{r} s_{k-1}^{r} v \quad \text { by (17) } \\
& =s_{i}^{r} s_{k-1}^{r} s_{j}^{q} v=s_{i}^{r} s_{k-1}^{r} y
\end{aligned}
$$

as required.

For property (18), the left side is $d_{i}^{p} s_{j}^{q} s_{k}^{r} w$, and the right side simplifies to this, using (18) and (19) when $p \neq q$, (16) when $p=q$ and $i$ is $j$ or $j+1$, and (18), (19) and (14) (resp., (15)) when $p=q$ and $i<j$ (resp., $i>j+1$ ).

For property (19), we want to know (using Remark 5.4) that $s_{k}^{r} s_{i}^{p} y=s_{i}^{p} s_{k}^{r} y$ when $r>p, y \in X_{\mathbf{n}-\mathbf{e}_{p}}$, and $s_{i}^{p} y$ is in the image of $s_{j}^{q}$ for some $q<r$. Choose the smallest such pair $(q, j)$; then $s_{i}^{p} y=s_{j}^{q} w$ for some $w$. If $p \neq q$ the result follows easily from Lemma 6.1 and (19); if $p=q$ the proof is similar to that for (5) in Section 2. 


\section{Proof of Theorem 5.3: Case 2}

Choose the smallest $j$ for which $x$ is in the image of $s_{j}^{r}$; then

$$
x=s_{j}^{r} w
$$

for a unique $w$. Define

$$
s_{k}^{r} x=s_{j}^{r} s_{k-1}^{r} w
$$

(as required by (17)). We need to verify (14)-(18); (19) is not relevant for this Case.

The proofs of (14)-(17) are essentially the same as the proofs of (2)-(5) in Section 2.

For (18), both sides simplify to $s_{j}^{r} s_{k-1}^{r} d_{i}^{p} w$, using (18) on the left and (18) and (17) on the right.

\section{Proof of Theorem 5.3: Case 3}

The argument for this Case is similar to that given in Sections 3 and 4.

Lemma 8.1. Let $X$ be an $\ell$-fold multisemisimplicial set satisfying the Kan condition. Then there are functions

$$
T_{j}^{q}: X_{\mathbf{n}} \rightarrow X_{\mathbf{n}+2 \mathbf{e}_{q}}
$$

for $0 \leqslant j \leqslant n_{q}$, with the following properties.

$$
\begin{aligned}
d_{i}^{q} T_{j}^{q} & =T_{j-1}^{q} d_{i-1}^{q} & & \text { if } 0<i<j+1 . \\
d_{i}^{q} T_{j}^{q} & =T_{j}^{q} d_{i-2}^{q} & & \text { if } i>j+2 . \\
d_{j+1}^{q} T_{j}^{q} & =d_{j+2}^{q} T_{j}^{q} & & \text { for all } j . \\
d_{0}^{q} d_{j+1}^{q} T_{j}^{q} x & =x & & \text { for all } j \text { and } x . \\
d_{i}^{p} T_{j}^{q} & =T_{j}^{q} d_{i}^{p} & & \text { whenever } p \neq q .
\end{aligned}
$$

Assuming the lemma, we complete the proof of Theorem 5.3. Given that $x$ is not in the image of $s_{j}^{q}$ for any pair $(q, j)<(r, k)$, we define

$$
s_{k}^{r} x=d_{0}^{r} T_{j}^{r} x
$$

The proofs of (14)-(16) are the same as the proofs of (2)-(4) at the end of Section 3, and (17) is not relevant for this Case. Property (18) is immediate from (24), and (19) is not relevant for this Case.

It remains to prove Lemma 8.1. The proof is similar to that of Lemma 3.1 in Section 4.

First we define $y_{j}^{q}$ for $(q, j) \neq(r, k+1)$ by

$$
y_{j}^{q}= \begin{cases}x & \text { if } q=r \text { and } j=0, \\ d_{k}^{r} T_{k-1}^{r} d_{j-1}^{r} x & \text { if } q=r \text { and } 0<j<k+1, \\ d_{k+1}^{r} T_{k}^{r} d_{j-1}^{r} x & \text { if } q=r \text { and } j>k+1, \\ d_{k+1}^{r} T_{k}^{r} d_{j}^{q} x & \text { if } q \neq r .\end{cases}
$$

The verification that these are consistent is a routine modification of the argument in Section 4 and is left to the reader.

The Kan condition gives an element $y$ with $d_{j}^{q} y=y_{j}^{q}$ for $(q, j) \neq(r, k+1)$. 
Now define $z_{j}^{q}$ for $(q, j) \neq(r, 0)$ by

$$
z_{j}^{q}= \begin{cases}T_{k-1}^{r} d_{j-1}^{r} x & \text { if } q=r \text { and } 0<j<k+1, \\ y & \text { if } q=r \text { and } j \text { is } k+1 \text { or } k+2, \\ T_{k}^{r} d_{j-2}^{r} x & \text { if } q=r \text { and } j>k+2, \\ T_{k}^{r} d_{j}^{q} x & \text { if } q \neq r .\end{cases}
$$

Again, the verification that these are consistent is a routine modification of the corresponding argument in Section 4.

Now the Kan condition gives an element $T_{k}^{r} x$ with $d_{j}^{q} T_{k}^{r} x=z_{j}^{q}$, and properties (20)-(24) are immediate from the construction.

\section{A result of Rourke and Sanderson}

As in [4], we will use $\mid$ for the geometric realization of a semisimplicial set and | $\left.\right|_{M}$ for the geometric realization of a simplicial set (the M stands for Milnor).

The following result is Corollary 5.4 of [4].

Proposition 9.1. Let $Z \rightarrow W$ be a semisimplicial inclusion with the property that $|Z|$ is a retract of $|W|$. Let $X$ be a semisimiplicial set satisfying the Kan condition, and let $f: Z \rightarrow X$ be any semisimplicial map. Then $f$ extends to $W$.

The rest of this section gives a new proof of this result.

First we need some notation. The singular complex functor from topological spaces to simplicial sets will be denoted by $S$. As in [4], the free functor from semisimplicial to simplicial sets will be denoted by $G$; recall that an element of $(G Y)_{n}$ is a pair $(\lambda, y)$, where $y \in Y_{p}$ for some $p \leqslant n$ and $\lambda: \Delta^{n} \rightarrow \Delta^{p}$ is a degeneracy or (if $p=n$ ) the identity map.

There is a natural map of semisimplicial sets

$$
\alpha: Y \rightarrow S|Y|
$$

which takes $y \in Y_{n}$ to the function $h_{y}: \Delta^{n} \rightarrow|Y|$ defined by $h_{y}(u)=[u, y]$ (where $[u, y]$ denotes the class of $(u, y)$ in $|Y|)$. This extends to a natural map of simplicial sets

$$
\bar{\alpha}: G Y \rightarrow S|Y|
$$

Lemma 9.2. $\bar{\alpha}$ is a trivial cofibration.

Proof of Lemma 9.2. Recall that a cofibration of simplicial sets is a 1-1 map. To see that $\bar{\alpha}$ is a cofibration, suppose that $\bar{\alpha}(\lambda, y)=\bar{\alpha}\left(\lambda^{\prime}, y^{\prime}\right)$. Then $[\lambda(u), y]=\left[\lambda^{\prime}(u), y^{\prime}\right]$ for every $u$ in the relevant $\Delta^{n}$. Choosing $u$ to be an interior point, we see that $y=y^{\prime}$. It follows that $\lambda=\lambda^{\prime}$ on the interior of $\Delta^{n}$, and hence, by continuity, on all of $\Delta^{n}$.

Next we must show that $\bar{\alpha}$ induces a weak equivalence of realizations. For this it suffices to note that the following diagram commutes:

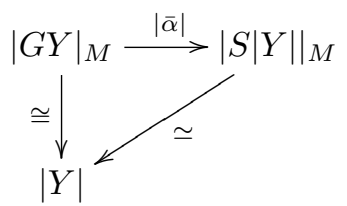


Now let $Z, W, X$ and $f$ be as in the statement of Proposition 9.1. Use Theorem 1.2 to give $X$ a compatible simplicial structure. Then the map $f: Z \rightarrow X$ extends to a map $\bar{f}: G Z \rightarrow X$ of simplicial sets. Since $\bar{\alpha}$ is a trivial cofibration of simplicial sets and $X$ is a Kan simplicial set, there is a map $\phi: S|Z| \rightarrow X$ with $\phi \circ \bar{\alpha}=\bar{f}$.

Next let $r:|W| \rightarrow|Z|$ be a retraction and let $g: W \rightarrow X$ be the composite

$$
W \rightarrow G W \stackrel{\bar{\alpha}}{\rightarrow} S|W| \stackrel{S r}{\rightarrow} S|Z| \stackrel{\phi}{\rightarrow} X .
$$

The commutativity of the following diagram shows that $g$ restricts to $f$ as required.

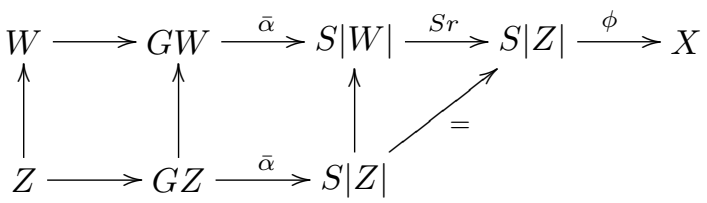

\section{References}

[1] Samuel Eilenberg and J. A. Zilber, Semi-simplicial complexes and singular homology, Ann. of Math. (2) $\mathbf{5 1}$ (1950), 499-513.

[2] Gerd Laures and James McClure, Commutativity properties of Quinn spectra, in preparation.

[3] A. A. Ranicki, Algebraic L-theory and topological manifolds, Cambridge Tracts in Mathematics, vol. 102, Cambridge University Press, Cambridge, 1992.

[4] C. P. Rourke and B. J. Sanderson, $\triangle$-sets. I. Homotopy theory, Quart. J. Math. Oxford Ser. (2) 22 (1971), 321-338.

[5] Stefan Schwede, Topological triangulated categories, preprint available at http://arxiv.org/abs/1201.0899.

[6] Charles A. Weibel, An introduction to homological algebra, Cambridge studies in advanced mathematics, Cambridge University Press, Cambridge, 1994.

James E. McClure mcclure@math.purdue.edu

Dept. of Mathematics, Purdue University, 150 N. University St., West Lafayette, IN, 47907-2067, USA 